\title{
Lay Summaries of Clinical Study Results: An Overview
}

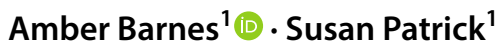

Published online: 14 June 2019

(c) The Author(s) 2019

\begin{abstract}
The European Union Clinical Trials Regulation (EU CTR) 536/2014 includes a requirement for the submission of lay summaries. Study participants, advocacy groups, and, to a lesser extent, the general public have called for greater transparency in their quest for information on clinical studies. As a complement to other forms of clinical study disclosure such as registry postings and scientific publications, lay summaries may aid in the transparency of a sponsor's clinical study results, thereby promoting trust, partnership, and patient engagement throughout the clinical study process. The data transparency field is changing rapidly; therefore, data owners should strive to stay abreast of the changes and deliver meaningful tools to their study participants and the public. Points to consider when developing lay summaries of clinical study results include regulatory drivers, the target audience, communication of complex data in a lay manner, and efficient processes for the development of lay summaries within one's company.
\end{abstract}

\section{Plain Language Summary}

There is a rule in Europe that clinical studies (experiments in humans) must have a summary written in plain language. Summaries written in plain language help people who are not scientists or doctors understand complex medical information. People who participate in clinical studies, and others, may want to know information about clinical study results. Lay summaries are a way to share clinical study results, but they do not replace other ways that information is shared. Lay summary writers must think about how they can help readers understand the information. It is hard to describe the results of clinical studies in a way that everyone can understand. This article gives some ideas to think about when writing lay summaries.

\section{Key Points}

Lay summaries of clinical study results are a complement to other forms of clinical study disclosure that aid in the understanding of complex clinical study results.

The initial requirement for lay summaries began as a result of the EU Clinical Trial Regulation; however, evolving regulations and policies around the globe are shaping the future of clinical study disclosure.

Ensuring patient value should be of paramount importance when developing lay summaries.

Amber Barnes

amber.barnes@ucb.com

1 UCB Bioscience, RTP, 8010 Arco Corporate Drive, Raleigh, NC 27617, USA

\section{What are Lay Summaries and Why are They Needed?}

Lay summaries (also called layperson summaries, plain language summaries, lay language summaries, simple summaries, and trial results summaries) are plain language descriptions of the design and aggregate results of individual clinical studies (Fig. 1).

Lay summaries are one way for industry to provide greater transparency to those interested in learning about clinical study results [2]. These documents are written specifically for study participants and the general public who have an interest in clinical study results, but who may have limited health literacy or scientific expertise. Health literacy is defined as the degree to which individuals have the capacity to obtain, process, and understand basic health information and services needed to make appropriate health decisions [3]. The goal of a lay summary is to aid study participants and the general public in 

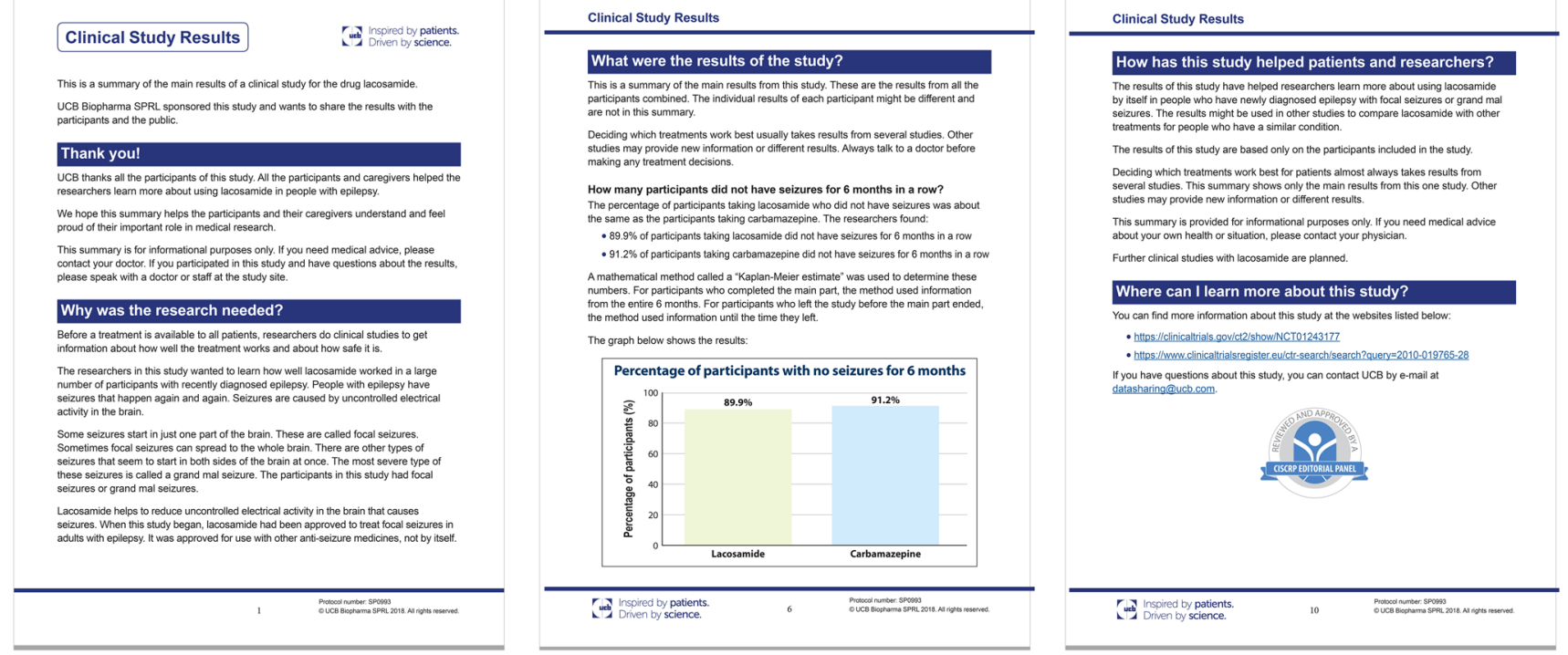

Fig. 1 Excerpted pages from a published lay summary [1]

understanding clinical study results. Not only does this effort help to demystify the clinical study process but it also provides the main results of clinical studies in a manner directed specifically towards people with low health literacy. The target audience is any person interested in research, and, as such, the audience is broad and may include study participants, healthcare professionals, caregivers, and the general public.

The data transparency field is changing rapidly, with recognition that the desires of patients and the general public may go beyond those driven by regulatory requirements. Although regulatory agencies do not currently require submission of lay summaries, some agencies have provided additional statements on the topic, as summarized below, in preparation for when the regulations come into effect. The commitment of a sponsor company to provide lay summaries in advance of regulatory requirements provides a meaningful way of communicating respect for the needs and desires of the community, particularly study participants.

The push for increased transparency in the clinical study space has presented industry and academia with an interesting choice - to proactively engage or only meet regulatory requirements. Currently, some transparency activities are not required per regulations and are implemented solely based on a company's own policies on clinical transparency and data sharing. For example, the proactive release of clinical documents and datasets can occur through various mechanisms such as portals (e.g. clinicalstudydatarequest.com, yoda.yale.edu), registration and results disclosure (e.g. clinicaltrials.gov), or posting lay summaries of clinical study results to publicly available websites. Other transparency activities are regulatory driven, such as the release of redacted or anonymized documents for European Medicines Agency (EMA) Policy 0070.

\section{What are the Current Regulatory Requirements?}

\subsection{European Medicines Agency: EU Clinical Trial Regulation 536/2014}

The EMA initiated the call for the submission of lay summaries through the European Union Clinical Trials Regulation (EU CTR) 536/2014. Among other elements described in the regulation, sponsors must submit a summary of the results of the clinical study, together with a summary and the Clinical Study Report (CSR), irrespective of the outcome of the study. The summary must be provided in a 'format understandable to laypersons', with posting to the portal 1 year after the end of the trial (EoT) for studies in adults, and 6 months after the EoT for studies in the pediatric population, in all the EU languages in which the study was conducted (Fig. 2).

Although this regulation entered into force on 16 June 2014, the timing of its application depends on the development of a fully functional EU clinical trials portal and database. In October 2018, the EMA reported that the development of the auditable release of the portal and database was complete, and then began the extensive phase of pretesting that must occur before formal user acceptance testing in early 2019. The EMA noted that some delay was anticipated due to the EMA's move to Amsterdam. Currently, the EMA estimates a system go-live date later in 2020 [4]. 


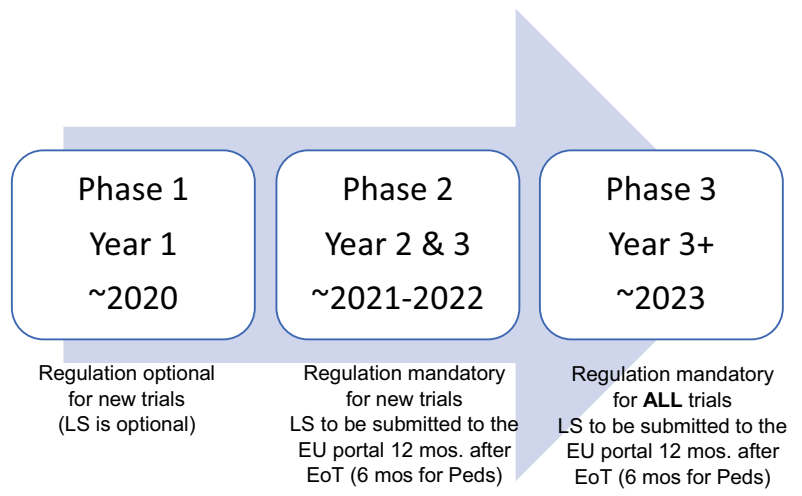

Fig. 2 EU Clinical Trial Regulation 536/2014potential timeline. EoT end of trial (defined as the last visit of the last participant in all concerned member states, or at a later point in time as defined in the protocol), $L S$ lay summary, Peds pediatric patients, mos months

Once the regulation is in application, all new interventional clinical studies will need to comply; however, the regulation includes three phases of implementation. Assuming the dates currently projected by the EMA, this would provide the following timescale for sponsors to submit lay summaries:

Phase 1: A 1-year introductory period where regulations are optional.

Phase 2: Spanning the second and third year of the transitory period; the regulation will be mandatory for new studies.

Phase 3: After the 3-year transitory period described above, the regulation will be mandatory for all studies (both ongoing and new studies).

Figure 3 is an example of the timeline described by the EMA above, assuming the EU CTR comes into application on 1 July 2020 (arbitrary date chosen for illustrative purposes). Company A chooses to comply with the regulation during Phase 1. On 1 July 2020, Company A submits a Clinical Trial Application (CTA) under the new regulation and the EoT date is 6 months later, on 1 January 2021. Company A submits lay summaries on 1 January 2022, approximately 1.5 years after the regulation comes into application.

Conversely, Company B has a lengthy clinical study and chooses not to participate in Phase 1. On 1 July 2021, Company B submits a CTA under the new regulation. The EoT date is not until 1 January 2023. Company B would not be required to submit a lay summary until 1 January 2024, approximately 2.5 years after the EU CTR comes into application. Continued delays to the application date of the EU CTR, combined with sponsors not participating in Phase 1, will exacerbate the demand by study participants, patient advocates, and other interested persons for the public release of these important documents.

\subsection{The Netherlands: Medical Research Involving Human Subjects Act}

In 2015, the Dutch Ethics Committee (Central Committee on Research Involving Human Subjects [CCMO]) implemented new transparency rules for CTAs [5]. The public disclosure of study results in the CCMO register applies only to new CTAs submitted as of 15 December 2015. The requirement states that a scientific summary of the study results should be submitted to the committee who reviewed the study (accredited Medical Research Ethics Committee [MREC] or CCMO). These summaries must be submitted via the webportal ToetsingOnline within 12 months of the end of the study worldwide. Although a public summary is required, the regulation does not clearly state if it is to be a lay summary. However, the User Manual for Toetsing Online

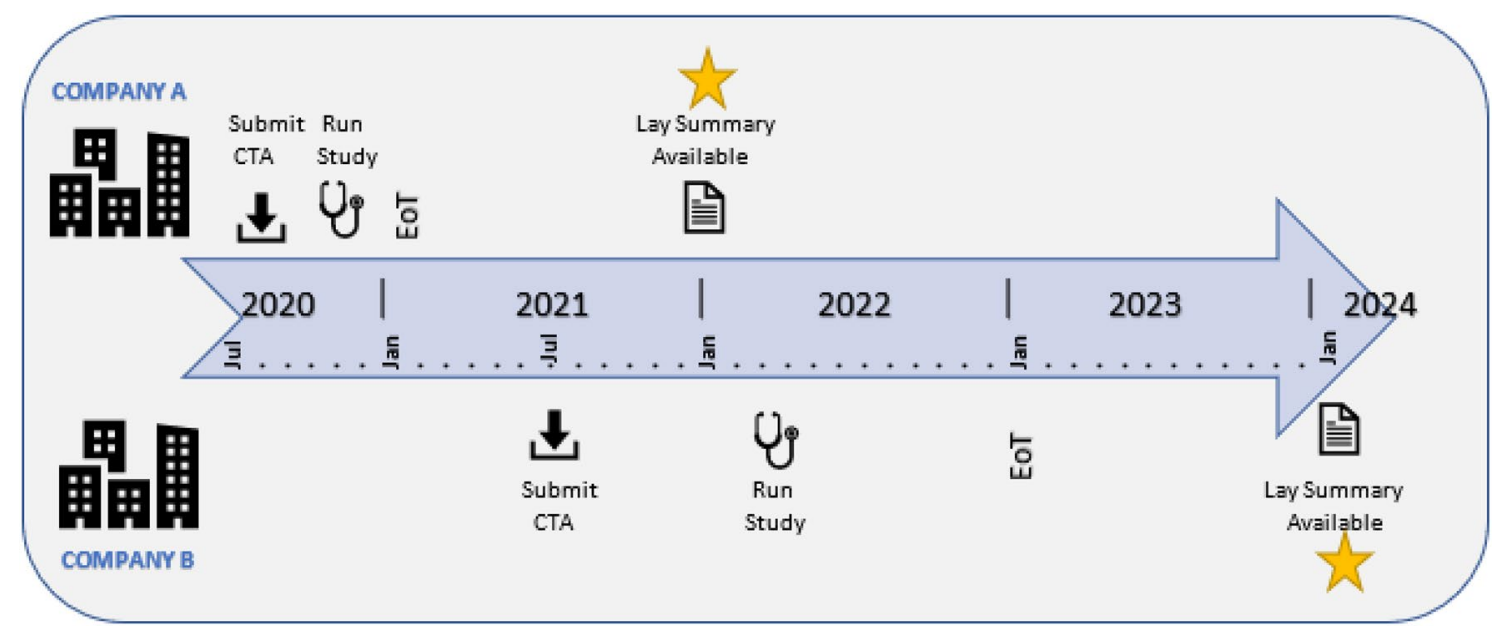

Fig. 3 Example of timing differences for the disclosure of lay summaries. CTA clinical trial application, EOT end of trial 
states "This final report will consist of a lay summary and a scientific summary with study results. This has to be submitted via the webportal ToetsingOnline. It is also possible to refer to a public website where the study results are published. The public disclosure will take place in the CCMO register." Additionally, it is noted that a sponsor can refer to a public website where the results have been published. Industry guidance on Dutch requirements will continue to evolve as more sponsors gain experience with this regulation and the requirements for results disclosure.

\subsection{United States: Food and Drug Administration Clinical Trial Transparency Pilot Program}

In January 2018, Scott Gottlieb, MD, who was at that time the US FDA Commissioner, released a statement indicating that the FDA planned to pursue a pilot program to evaluate whether disclosing certain information included within CSRs following approval of a New Drug Application (NDA) improves public access to drug approval information [6]. In this pilot, the FDA chose up to nine recently approved NDAs whose sponsors were selected to participate. The FDA posted portions of pivotal clinical study-related summaries submitted on Drugs@FDA. As part of this effort, the FDA intended to post the parts of the CSRs that were most important to the FDA's assessment of the safety and efficacy of the drug. Specifically, they included the study report body, the protocol and amendments, and the statistical analysis plan for each of the participating product's pivotal studies.

Although there is currently no FDA regulation requiring lay summaries of clinical trial results to be publicly disclosed, there are indications of movement in the US research environment that may signal future requirements are on the horizon. For instance, the National Institutes of Health (NIH) has stated a desire for "broad, transparent, timely, and responsible dissemination of information on NIH-funded clinical trials" [7]. This, coupled with the Plain Writing Act of 2010, which requires the use of plain language for information disseminated to the general public, and the Final Rule, which requires posting of informed consent forms (ICF) for trials overseen by the FDA that have a federal department or agency conducting the study, is further indication that the US may be moving towards a broader implementation of disclosing clinical study information in plain language to the general public $[8,9]$.

\subsection{Canada: Health Canada Public Release of Clinical Information}

On 13 March 2019 Health Canada announced the implementation of the Public Release of Clinical Information regulation [10], which states that clinical information on drugs and medical devices will be made available to Canadians through
Health Canada's new Clinical Information Portal. In this regulation, Health Canada stated that certain clinical information contained in drug submissions (Clinical Overviews and Clinical Summaries within Module 2, and CSRs within Module 5) and medical device applications (in Chapter 4 for Class III and IV medical devices; Class I and II medical device applications and amendments are out of scope) will be made publicly available for 'non-commercial' purposes following regulatory review. The regulation has a retroactive scope effective immediately for both drugs and devices; however, proactive release of device information has been deferred until 2021. There is no requirement to submit lay summaries to Health Canada as a part of their transparency efforts.

\section{What is the Content of a Lay Summary?}

Annex V of the EU CTR specifies the 10 essential elements that must be included in a lay summary. The content of lay summaries has evolved over the past 5 years, with earlier versions not always including all the elements now required. An example of lay-friendly headings for the required elements are presented in Table 1.

\section{What to Consider When Creating a Lay Summary}

As with any effective communication, the needs of the intended audience must be a primary consideration in lay summary development. Since lay summaries are created for the general public, there may be considerable variation in scientific knowledge, general and health literacy levels, and numeracy skills. Per the EU CTR guidance, readability should be targeted at a literacy proficiency level of 2-3 on the International Adult Literacy Survey scale. A wellwritten lay summary should be understandable to people 12 years of age and older [11]. While it is not difficult to achieve an appropriate literacy level based on word-, syllable-, and sentence count-based formulae, a more challenging target is achieving an appropriate readability level for health literacy. A World Health Organization (WHO) 2013 health literacy study found that $48 \%$ of adults have problematic or inadequate health literacy. This underscores the importance of considering health literacy when authoring lay summaries of clinical study results [12].

To maximize the incorporation of health literacy considerations into lay summaries, it is essential to include study participants and people who may have limited scientific knowledge and health literacy in the creation of lay summaries. Ideally, input from these contributors should occur during development of the lay summary template to be used across studies and once the template is populated with results 
Table 1 Lay-friendly headings for required elements of a lay summary

\begin{tabular}{|c|c|}
\hline Required element & Lay-friendly heading \\
\hline $\begin{array}{l}\text { Clinical trial identification (including title of the trial, protocol number, EU trial number, } \\
\text { and other identifiers) }\end{array}$ & Study information \\
\hline Name and contact details of the sponsor & Where can I learn more about this study? \\
\hline $\begin{array}{l}\text { General information about the clinical trial (including where and when the trial was con- } \\
\text { ducted, the main objectives of the trial, and an explanation of the reasons for conducting } \\
\text { the trial) }\end{array}$ & $\begin{array}{l}\text { Why was the research needed? } \\
\text { What were the main questions studied? }\end{array}$ \\
\hline $\begin{array}{l}\text { Population of subjects (including information on the number of subjects included in the } \\
\text { trial in the Member State concerned, in the Union, and in third countries; age group } \\
\text { breakdown and gender breakdown; inclusion and exclusion criteria) }\end{array}$ & Who participated in the study? \\
\hline Investigational medicinal products used & What treatments did the participants take? \\
\hline Description of adverse reactions and their frequency & What medical problems did the participants have? \\
\hline Overall results of the clinical trial & $\begin{array}{l}\text { What happened during the study? } \\
\text { What were the results of the study? }\end{array}$ \\
\hline Comments on the outcome of the clinical trial & How has this study helped patients and researchers? \\
\hline Indication if follow-up clinical trials are foreseen & $\begin{array}{l}\text { Within the "How has this study helped patients and } \\
\text { researchers?" section }\end{array}$ \\
\hline Indication where additional information could be found & Where can I learn more about this study? \\
\hline
\end{tabular}

$E U$ European Union

for an individual study. For lay summaries of pediatric studies, the additional participation of children and caregivers in the development of lay summaries is critical. The EU CTR guidance advises reader testing when feasible [4]. Additionally, a recent study by Raynor et al. demonstrated that user testing can be used to improve the readability and acceptability of lay summaries to the non-medical public [13].

Various models exist to obtain study participant and general public feedback on lay summaries, including hosting within the sponsor company, partnering with an independent group, or utilizing online services to host virtual panels. The authors have found that feedback from study participants and/or lay panels is invaluable to the success of lay summary development.

Consistency of study descriptions and terminology between the ICF and the lay summary for each study is likely important for easing study participants' comprehension of lay summaries as the reader is presented material in a consistent fashion. In addition to the benefit for study participants, transportability of text from the ICF to the lay summary increases lay summary authoring efficiency.

\section{What are Some Challenges of Lay Summary Authoring?}

\subsection{Adverse Drug Reactions and Overall Results}

After publication of the EU CTR, expert group recommendations were published to provide additional clarification of the Annex V requirements [11]. This guidance included clarification of Item 6 (Description of Adverse Reactions and Their Frequency) and Item 7 (Overall Results of the Clinical Study), as summarized below.

\subsubsection{Description of Adverse Reactions and Their Frequency}

The expert group acknowledged the difficulty of defining adverse drug reactions in a single study, and recommended inclusion of adverse events considered related to the study drug as meeting the intent of the requirement. While the expert group recommended consideration be given to the use of 'side effects' to describe drug-related adverse events, for many sponsors this leaves open the possibility that the reader may confuse drug-related adverse events captured at a study level with adverse drug reactions captured at the investigational product level, typically based on more extensive databases and described in the product labeling. Therefore, use of the term 'adverse reaction' rather than 'side effect' is preferred by some sponsors and non-profit lay summary experts as a means of reducing possible confusion between study-level and investigational product-level designations. Regardless of what term is chosen, the term should be clearly defined in the lay summary.

Health literacy challenges are implicit in the presentation of adverse reactions. Technical documents typically include summaries of adverse event data using the Medical Dictionary for Regulatory Activities (MedDRA) Preferred Terms (PTs). Plain language descriptions of the PTs, which are often medical terms unknown to lay persons, must not only be accurate to the hierarchy applied by MedDRA but also be 


\section{Glossary}

\begin{tabular}{|l|l|}
\hline $\begin{array}{l}\text { Increase of a protein called gamma } \\
\text { glutamyltransferase: }\end{array}$ & $\begin{array}{l}\text { Gamma glutamyltransferase is made by the liver } \\
\text { and other parts of the body. An increase is also } \\
\text { called "gamma glutamyltransferase increased." }\end{array}$ \\
\hline $\begin{array}{l}\text { Seizure that starts in one side of the } \\
\text { brain and spreads: }\end{array}$ & $\begin{array}{l}\text { Also called "partial seizures with secondary } \\
\text { generalization." }\end{array}$ \\
\hline Severe drug reaction called DRESS: & $\begin{array}{l}\text { DRESS stands for "drug reaction with eosinophilia } \\
\text { and systemic symptoms." It is a reaction to a drug } \\
\text { that may include rash, fever, swollen lymph nodes, } \\
\text { and an increase in blood cells called eosinophilia. } \\
\text { It may also cause problems with some internal } \\
\text { organs. }\end{array}$ \\
\hline Sleepiness: & Also called "somnolence." \\
\hline Tiredness: & Also called "fatigue." \\
\hline
\end{tabular}

Fig. 4 Example of a glossary of terms from a published lay summary [1]

sufficiently succinct for presentation in lay summary displays. Consistency is important, therefore a master lexicon of terms should be developed. Figure 4 provides an example of lexicon entries developed by an internal plain language expert within a sponsor company and agreed upon by the study team for use in lay summaries. In this figure, the MedDRA PTs are provided, along with the sample plain language descriptions. Use of a lexicon not only promotes consistency across lay summaries but also contributes to resource savings by reducing writing time and translation time.

\subsubsection{Overall Results of the Clinical Study}

The expert group recommends including a general statement on the overall study results and presentation of primary endpoints prespecified in the statistical design of the study [11]. Primary endpoints should be presented by treatment group, as applicable, with a lay-friendly statement regarding the statistical relevance of the results. All the endpoints may be of interest to readers, however the sheer number of endpoints often precludes presentation. By presenting only primary endpoints, the sponsor avoids the possible promotional concern of being perceived as selecting only the more favorable endpoints for presentation, or inconsistency in presentations across assets. All publicly available results (e.g. clinicaltrials. gov and clinicaltrialsregister.eu) should be referenced in the lay summary, therefore the interested reader will have access to all non-primary endpoints through these other databases.

\subsection{Achieving a Non-Promotional Tone}

The goal of achieving a non-promotional tone is essential to the development of fit-for-purpose lay summaries. To create a fair and balanced presentation, text and graphical presentations must be factual and objective. Similarly, the organization of the lay summary should in no way highlight positive outcomes while minimizing negative outcomes. While the use of graphics can help in reader understanding, careful consideration must be given to the presentation itself, including coloration. Simplified graphics should be included only if their intent is to support understanding and not simply to increase visual appeal. All lay summaries should strike a balance between factual text and useful graphics to aid in the reader's understanding. Authors must pay particular attention to the presentation of comparisons, avoiding the use of superlatives (e.g. 'better' or 'worse') and claims (e.g. 'the results proved'), and be attentive to the limitations of the study design [14]. Finally, the use of percentage cut-offs for presentation of some data (e.g. adverse reactions) across similar-sized studies may provide for uniformity across the sponsor's portfolio.

Sponsors may choose other means to share the lay summaries with the general public (e.g. websites, directly to study participants where allowed by law), however the potential for possible consideration as promotional material needs to be carefully considered. If a lay summary is published on a website, sponsors should ensure there is no link between promotional webpages and a page hosting lay summaries. If the sponsor intends to share the lay summaries with study participants, the timing, impact on ICF content, method, Institutional Review Board involvement, and possible impact on site closure and final payment needs to be carefully considered. Additional safeguards to ensure a non-promotional tone include making a clear statement in the document that the results presented are specific to a single study and no changes to a study participant's care plan should be made based on the results provided in the lay summaries. TransCelerate Biopharma Inc. has provided guidance to industry on the need to provide factual, objective, and non-promotional lay summaries [15].

\subsection{Translation of Lay Summaries}

The translation of lay summaries is another important consideration as the EU CTR requires that a translated version be submitted in the language of each EU country in which the study was conducted. In practice, lay summaries should be translated into all languages in which the ICF was created. It is important to be aware of the potential to introduce content differences between the English-language lay summary and the translated summaries.

Use of colloquial language, which is sometimes needed for effective communication of information to a lay audience, is a particular challenge for translation. To help ensure the fidelity of translations, sponsors should obtain a backtranslation certification for any translated lay summaries. In addition, translation is an important consideration in timeline planning, especially, but not exclusively, with respect to 
the shortened timelines for submission of lay summaries for pediatric studies, which have a '6-month from EoT' requirement for submission.

\subsection{Dissemination of Lay Summaries}

As shown by Sood et al, approximately $90 \%$ of people want to know the results of the clinical study in which they participated [16]. There are various options for the dissemination of lay summaries to study participants or the general public. As presented above, sponsors may choose to share lay summaries on a publicly available website. This is a non-targeted approach that potentially allows for the greatest exposure to the lay summary; however, it does not allow for an interactive experience for the end user. Sponsors may want to consider dissemination directly to study participants through the investigative site. This form of dissemination provides an opportunity for participants to discuss results directly with the investigator, ideally promoting patient engagement and enhanced understanding of clinical studies. Another option for dissemination to study participants is via direct mailing using a third-party vendor.

\section{How Can You Ensure an Efficient Process?}

\subsection{Promote Organizational Awareness and Training for Reviewers and Approvers}

The preparation of plain language descriptions is a new and often challenging activity for project teams. Traditionally, study teams are typically focused on design and implementation of clinical studies and preparation of scientifically and statistically dense documents targeted for regulatory or peer audiences. Often, those who have the expertise to comprehend the scope, nuances, and limitations of clinical study design are challenged to communicate without the use of entrenched clinical study and statistical jargon that holds little meaning for the lay audience. Since these subject matter experts will likely be involved in the review of lay summaries, it is important that training is provided to team members on lay language communication.

A successful educational effort should acknowledge the challenge team members will face in balancing scientific depth with information directed at a lay audience. General awareness regarding lay summaries should be available to the entire organization, while more intensive training efforts should be directed at reviewers and approvers of the lay summaries. As in all learning, repetition of core considerations (general literacy, health literacy, numeracy, people focus, and non-promotional tone) and frequent reminders of the target audience is essential. Reviewers and approvers will vary depending on organizational structure, but representatives from legal, clinical, statistics, pharmacovigilance, results disclosure, and regulatory should be considered.

\subsection{Plan Within Your Clinical Study Report Development Timeline}

Ideally, all non-data-dependent sections of the lay summary should be developed in parallel with the ICF and then finalized during the preliminary stages of CSR preparation. Providing text and graphic descriptions common to both documents will foster understanding of the lay summaries among study participants.

When the CSR text is in early development, lay summary displays and presentations should be readied for population with final data, once available. Thus, it is possible to have lay summary approval concurrent with CSR approval, minimizing time delays. While efficient preparation is important for all lay summaries, it is of key importance for lay summaries of pediatric studies that have a requirement for submission of no more than 6 months after the last participant's last visit.

\section{What is the Future of Lay Summaries?}

As the desire for lay-friendly documents grows in the public domain, the way companies communicate scientific and medical information will evolve. The use of creative tools, such as infographics or pictorial representations of data, are essential (Fig. 5). In the future, sponsors may need to consider reaching greater populations through novel approaches, such as video lay summaries. In addition, pediatric considerations, such as cartoons or comics, could become the norm,
Fig. 5 Targeted infographics can aid in readability [1]

\begin{tabular}{|l|l|}
\hline $\begin{array}{l}444 \text { participants took } 200 \text { to } 600 \\
\text { milligrams of lacosamide each day. }\end{array}$ & $\begin{array}{l}442 \text { participants took } 400 \text { to } 1200 \\
\text { milligrams of carbamazepine each day. }\end{array}$ \\
\hline $\begin{array}{l}\text { Participants took lacosamide } \\
\text { twice each day for up to } 118 \text { weeks. }\end{array}$ & $\begin{array}{l}\text { Participants took carbamazepine } \\
\text { twice each day for up to } 118 \text { weeks. }\end{array}$ \\
\hline $\begin{array}{l}\text { Each pill of lacosamide had } 50 \text { or } \\
100 \text { milligrams of lacosamide. }\end{array}$ & $\begin{array}{l}\text { Each pill of carbamazepine had } 200 \\
\text { milligrams of carbamazepine. }\end{array}$ \\
\hline
\end{tabular}


although these may appear to be promotional and should be strictly assessed.

It is likely that regulatory agencies will continue to expand their guidance and regulations to encourage more patient-focused outputs from sponsors. As the momentum grows, study participants, advocates, caregivers, and the general public will increasingly have an appetite for disclosure from sponsor companies. Through engagement directly with the target audience, the study participant, and the general public, sponsors can gain valuable insights on the focus of lay summaries, while shaping their processes and ensuring readiness when regulations become applicable.

As the lay summary space evolves, the focus must remain on communicating clear, factual, easy to understand results to study participants and non-technical audiences. Providing such information also comes with the added responsibility of ensuring a study participant can access additional information in a timely manner (e.g. discuss with their primary healthcare professional or study investigator). As a complement to other forms of clinical study disclosure, such as registry postings and scientific publications, lay summaries aid in the transparency of a sponsor's clinical study results, thereby promoting trust, partnership, and patient engagement throughout the clinical study process.

Acknowledgements UCB Biosciences, Inc. has a business agreement with the Center for Information and Study on Clinical Research Participation (CISCRP) to partner in lay summary preparation. The excerpts of lay summaries presented in this manuscript were prepared under this agreement. The authors would like to thank Janice Block for her editorial review of this article.

\section{Compliance with Ethical Standards}

Funding No funding was used to assist in the preparation of this article. Open access was funded by UCB Biosciences, Inc.

Conflict of interest Amber Barnes and Susan Patrick are employees of UCB Biosciences, Inc. The opinions and ideas expressed in this article are personal and do not necessarily represent those of the company. Susan Patrick declares that she has no other conflicts of interest in this work, and Amber Barnes declares that she owns stock in UCB Biosciences, Inc., but has no other conflicts of interest in this work.

Open Access This article is distributed under the terms of the Creative Commons Attribution-NonCommercial 4.0 International License (http://creativecommons.org/licenses/by-nc/4.0/), which permits any noncommercial use, distribution, and reproduction in any medium, provided you give appropriate credit to the original author(s) and the source, provide a link to the Creative Commons license, and indicate if changes were made.

\section{References}

1. UCB S.A. SP0993 Study results lay summaries. 2019. https:// www.ucb.com/_up/ucb_com_clinical_studies/documents/sp099 3-lay\%20summary_source_linked_.pdf. Accessed 11 Apr 2019.

2. Center for Information \& Study on Clinical Research Participation (CISCRP). Perceptions and insights study. Boston: CISCRP; 2017. https://www.ciscrp.org/download/2017-perceptions-insig hts-study-the-participation-experience/? $\mathrm{wpdmdl}=8770 \&$ refre sh=5cf912571eafa1559827031. Accessed 20 Nov 2018.

3. US Department of Health and Human Services. Quick guide to health literacy. 2018. https://health.gov/communication/literacy/ quickguide/factsbasic.htm. Accessed 20 Nov 2018.

4. European Medicines Agency (EMA). Clinical trial regulation. 2018. https://www.ema.europa.eu/en/human-regulatory/researchdevelopment/clinical-trials/clinical-trial-regulation. Accessed 20 Nov 2018.

5. Central Committee on Research Involving Human Subjects (CCMO). Explanation of the general assessment and registration (ABR) form (version 15 December 2015). 2018. http://www. ccmo.nl/attachments/files/b1-abr-toelichting-15-dec-2015-eng. pdf. Accessed 10 Aug 2018.

6. US Food \& Drug Administration. Press announcements. 2018. https://www.fda.gov/newsevents/newsroom/pressannouncements/ ucm592566.htm. Accessed 20 Nov 2018.

7. National Institutes of Health: Office of Extramural. Why changes to clinical trial policies? https://grants.nih.gov/policy/clinical-trial s/why-changes.htm. Accessed 9 Apr 2019.

8. H.R.946-plain writing act of 2010. https://www.congress.gov/ bill/111th-congress/house-bill/946. Accessed 9 Apr 2019.

9. Federal Register. Clinical trials registration and results information submission. 2016. https://www.federalregister.gov/d/201622129. Accessed 9 Apr 2019.

10. Health Canada. Public release of clinical information regulation. 2019. https://www.canada.ca/en/health-canada/services/drughealth-product-review-approval/profile-public-release-clinicalinformation-guidance/document.html\#s2.1. Accessed 13 Mar 2019.

11. European Medicines Agency. Recommendations of the expert group on clinical trials for the implementation of Regulation (EU) No 536/2014 on clinical trials on medicinal products for human use, Version 2. Amsterdam: European Medicines Agency; 2017.

12. World Health Organization. Health literacy the solid facts. 2013. http://www.euro.who.int/_data/assets/pdf_file/0008/190655/ e96854.pdf. Accessed 20 Nov 2018.

13. Raynor D, Myers L, Blackwell K, et al. Clinical trial results summary for laypersons: a user testing study. Ther Innov Regul Sci. 2018;52(2):606-28.

14. Multi-Regional Clinical Trials Center of Harvard and Brigham and Women's Hospital (MRCT). MRCT return of results toolkitversion 22. Cambridge: MCRT; 2016.

15. TransCelerate Biopharma Inc. Recommendations for drafting non-promotional lay summaries of clinical trial results. 2015. http://www.transceleratebiopharmainc.com/wp-content/uploa ds/2015/04/TransCelerate-Non-Promotional-Language-Guide lines-v10-1.pdf. Accessed 10 Aug 2018.

16. Sood A, Prasad K, Chhatwani L, et al. Patients' attitudes and preferences about participation and recruitment strategies in clinical trials. Mayo Clin Proc. 2009;84(3):243-7. 